\title{
A Compact and Versatile Scanning Tunnelling Microscope with High Energy Resolution for Use in a ${ }^{3} \mathrm{He}$ Cryostat
}

\author{
C. Debuschewitz, F. Münstermann, V. Kunej, and E. Scheer \\ Fachbereich Physik, Universität Konstanz, Universitätsstraße 10 D-78464 Konstanz, Germany \\ E-mail: christian.debuschewitz@uni-konstanz.de
}

We present a simple design for a very-low temperature STM for the investigation of mesoscopic superconductors. The nonmagnetic microscope operates in a conventional ${ }^{3} \mathrm{He}$ cryostat at $T=240 \mathrm{mK}$ and in magnetic fields up to $B=1 T$. Efficient filtering of all electrical lines at base temperature results in a voltage resolution $\approx 20 \mu \mathrm{V}$, which has been tested by measuring the differential conductance of superconducting aluminium. The successful operation in magnetic field is demonstrated by spectroscopy on superconducting aluminium as well as by demonstrating atomic resolution of $H O P G$.

\section{MOTIVATION}

Up to now only a small number of scanning tunnelling microscopes (STM) world-wide are operating at temperatures below $1 \mathrm{~K}$, in high magnetic fields, and achieve high energy resolution $\Delta E<1 \mathrm{mV}$. Some of them combine UHV and very low temperatures ${ }^{1-5}$ while other designs operate in conventional cryostats. ${ }^{6-8}$ The STMs for non-UHV conditions are mostly specialized instruments for investigating heavy fermion superconductors ${ }^{9}$ or the spatial dependence of the superconducting proximity effect. ${ }^{10-12}$ However, a compact STM that combines very low temperatures, operation in magnetic field, and very high energy resolution with robustness, versatility and easy handling is still lacking. Our new setup that is described in the present article fulfills these requirements. It is very small, matching with most commercial low-temperature facilities, does not require elaborate vibration damping and uses only the very modest amount of seven cables. Furthermore, it is already designed for lower temperatures than presented here, is in principle UHV compatible and can be combined with more complex positioning systems. The special physical project, for which purpose our STM was designed, is to investigate the 
superconducting proximity effect, i.e. the mutual influence on the density of states (DOS) of a superconductor and a normal metal if both are in good electrical contact. ${ }^{13}$ Since we want to achieve quantitative agreement between experiments and theories we will concentrate on the superconductor aluminium, because it is the best known representative for BCS superconductivity. $^{14}$

The low transition temperature of aluminium $T_{\mathrm{c}}=1.2 \mathrm{~K}$ and its low energy gap demands an operation well below $T_{\mathrm{c}}$ in the $\mathrm{mK}$ range. The necessary temperature range ensures the desired high energy resolution as well. The low critical magnetic field of $B_{\mathrm{c}}=10 \mathrm{mT}$ requires nonmagnetic materials, as well as materials with high thermal conductivity and well adapted thermal expansion coefficients in order to reduce drift and thermal stress in the STM. A further requirement is a large scan range of several micrometers in order to be able to locate predefined superconductor-normal metal contacts on a superconducting substrate. Habenicht et al. ${ }^{15}$ developed a method to produce ensembles of small metallic islands with high and constant density and size distribution over an area of $1 \mathrm{~mm}^{2}$. The fabrication parameter can be varied such, that islands of diameter between $100 \mathrm{~nm}$ up to $2 \mu \mathrm{m}$ and average spacing of $1 \mu \mathrm{m}$ up to several micrometers can be produced. This guarantees that at least one island can be found within the scan range of the STM without any coarse positioning system. Last but not least the STM has to be small and compact to be less sensitive to external vibrations.

\section{EXPERIMENTAL SETUP}

\subsection{Cryogenic System}

We chose a conventional ${ }^{3} \mathrm{He}$ cryostat Heliox VL with sample in vacuum fabricated by Oxford Instruments. The cryostat has a $1 \mathrm{~K}$-stage, an internal vibration-free sorption pump for the ${ }^{3} \mathrm{He}$ circle and achieves a base temperature of $240 \mathrm{mK}$ with a cooling power of $40 \mu \mathrm{W}$ at $500 \mathrm{mK}$.

A superinsulated helium dewar avoids a nitrogen bath which would cause vibrations due to boiling. The dewar is equipped with a 3 -axis superconducting magnet. The $z$-direction delivers a magnetic field up to $B=1 \mathrm{~T}$ perpendicular to the sample plane while the two split coils for $x$ and $y$ directions generate a field up to $B=0.5 \mathrm{~T}$. This allows us to apply the magnetic field in a suitable direction to the sample in order to minimize demagnetization effects due to the geometry of the superconductor.

The system is mounted in the basement of the building and rests on four air-damping vibration feet, which have the maximum damping at the adjusted system weight. Vibrations of the pumping line for the $1 \mathrm{~K}$-stage 
are decoupled by a rubber pumping line which is fed through a sand-filled box.

\subsection{STM Head and Electronics}

We use a single-tube scanner which is simultaneously used for the tip coarse approach and scanning. In order to obtain a large scan range the scan tube ${ }^{16}$ has a length of 1 inch and a diameter of $1 / 4$ inch. To fulfill requirements for spectroscopy on superconductors with small critical fields, nonmagnetic silver-coated electrodes on the scanner are used. The tube is glued into a macor ${ }^{17}$ body in order to reduce thermal stress on the piezo tube during cool-down. ${ }^{18}$ For good thermalization all other parts are machined from copper. As shown by Mugele et al. ${ }^{19}$ but with a magnetic slip stick mechanism and Kleindiek and Herrmann et al., ${ }^{20}$ a very low-mass of movable parts results in a high resonance frequency. Following the idea of low-mass design we use a modified design of Renner et al. ${ }^{21}$ which has a low-mass slider that carries the tip. During the development of the system we tested different materials and designs of the slider in order to minimize the mass and thus maximize the resonance frequency of the STM for reducing vibrational cross-talk. The smallest model has a mass as small as $238 \mathrm{mg}$. The slider shown in Fig. 1 consists of two half cylinders, which are clamped onto two sapphire rods. A screw and the spring underneath are used to adjust the friction force between the carrier and sapphire rods to $0.3 \mathrm{~N}$. For some measurements a copper slider design comparable to Renner et al. ${ }^{21}$ of $350 \mathrm{mg}$ in weight has been used. Silver conducting paint is used to glue the tip onto the slider. The tip approach is realized by application of a sawtooth voltage pulse. Due to the low mass of the movable parts, the STM is rigid enough to be screwed directly onto the ${ }^{3} \mathrm{He}$ reservoir without additional damping, which ensures a good thermal coupling. A high-frequency shielded box around the STM avoids thermal radiation from the helium bath.

The wiring consists of unshielded, single and twisted manganin wires for the scan piezo and the STM heater. Semi-rigid stainless-steel coaxial cables $^{22}$ are used (from the top of the cryostat down to the ${ }^{3} \mathrm{He}$ reservoir) for applying the bias voltage and measuring the tunnelling current. In order to achieve the necessary small voltage-step size in the spectroscopic measurements, a voltage divider of $1 / 125$ is inserted into the biasvoltage line at the $1 \mathrm{~K}$-stage. All lines are radio-frequency filtered via copper-powder filters. Filters with a particularly high attenuation of $-60 \mathrm{~dB}$ above $1 \mathrm{GHz}$ are used for the bias and the current line. ${ }^{23-25}$ This attenuation would be sufficient to operate the STM in a dilution refrigerator for achieving effective electron temperatures as small as $65 \mathrm{mK}$. Below 


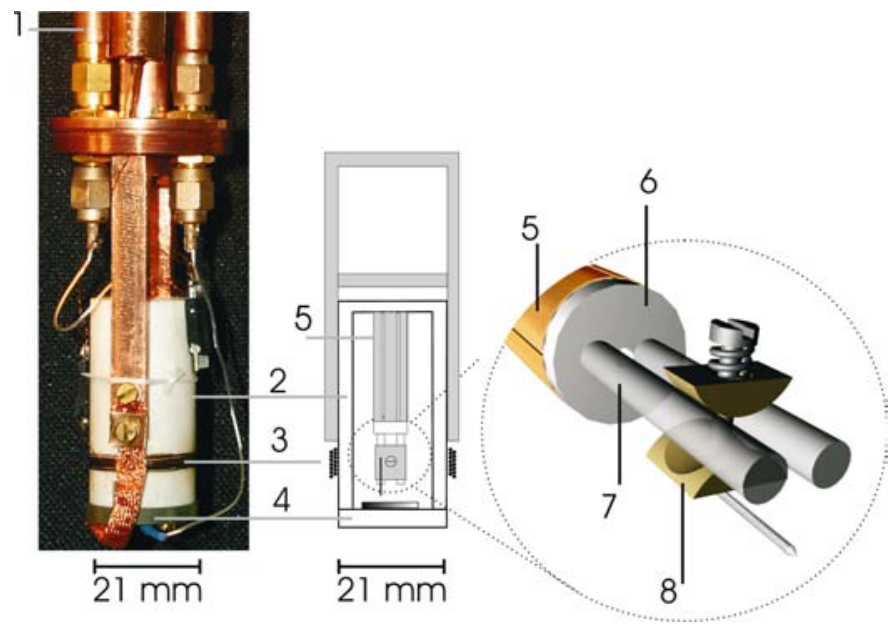

Fig. 1. (Color online) The photograph and the drawing in the centre show the whole STM setup with (1) copper-powder filters, (2) STM body made of macor, ${ }^{17}$ (3) heater, (4) sample holder and (5) the scan tube. The magnified drawing on the right hand side shows (5) the scan tube, (6) macor piece, (7) sapphire rods and (8) the copper slider with spring, screw and STM tip.

the filter stage all connections are made of copper wires and copper coaxial cables for optimal thermal contact. In order to reduce mechanical coupling between the STM body and the slider, the electrical connection is made by gluing a $25 \mu \mathrm{m}$ diameter gold wire onto the slider with silver conducting paint.

For controlling the STM we use the commercial RHK SPM 100 electronics with the $10^{9} \mathrm{~V} / \mathrm{A}$ preamplifier IVP-300 operating at room temperature with a cut-off-frequency of $15 \mathrm{kHz} .{ }^{26}$ The resulting noise caused by parasitic cable capacitance is about $150 \mathrm{fA} / \sqrt{\mathrm{Hz}}$ in a bandwidth of $5 \mathrm{kHz}$ (see below). For obtaining the maximum possible scan range we are using the $\pm 215 \mathrm{~V}$ scan-voltage option of the electronics.

For cooling down the system, the inner vacuum chamber is evacuated to a pressure of $5 \times 10^{-5}$ mbar before adding a small amount of exchange gas. In order to avoid cryosorption on the sample and the tip, a heater is wound around the macor body to keep the temperature of the STM approximately $30-60 \mathrm{~K}$ above the cryostat temperature during cool down from room temperature. The heater is switched off when the STM-body temperature is approximately $90 \mathrm{~K}$ and the rest of the cryostat is already below the freezing temperature of nitrogen. The tip approach is performed already at $T=1.7 \mathrm{~K}$ during condensation of the ${ }^{3} \mathrm{He}$ in order to minimize 
heat load when the system is at base temperature. Although not tested we assume the STM to be compatible with UHV conditions as well. A system identical in construction that works at $4 \mathrm{~K}$ is regularly baked at $150{ }^{\circ} \mathrm{C}$.

\section{CHARACTERIZATION OF THE SYSTEM}

\subsection{Results on a Superconducting Tip}

At $T<1 \mathrm{~K}$ effective thermalisation of the electronic system in transport measurements is a difficult task because of the long energy relaxation time and the coupling to the room temperature parts of the electronics. In superconductors in particular photons in the frequency range of several $\mathrm{GHz}$ corresponding to the typical excitation energies of quasiparticles within the superconducting energy gap are strongly affecting the observability of the superconducting density of states. On the other hand this fact can be used to determine the effective temperature $T_{\mathrm{el}}$ of the electron gas, which is achieved with the STM by measuring the differential conductance $d I / d V$ of a superconducting metal which is related to the DOS as described below. We measure the $d I / d V$ of aluminium with lock-in technique. The tunnelling current is a convolution of the (usually chosen normalconducting) probe DOS $\rho_{\mathrm{p}}$, the sample DOS $\rho_{\mathrm{s}}$ and the corresponding Fermi functions $f_{\mathrm{p}}$ and $f_{\mathrm{s}}$. The latter factors contain the electron temperature $T_{\mathrm{el}}$ which we wish to determine. ${ }^{27,28}$

$$
I(V) \propto \int_{-\infty}^{\infty} \rho_{\mathrm{s}}(E) \rho_{\mathrm{p}}(E+e V)\left[f_{\mathrm{s}}(E)-f_{\mathrm{p}}(E+e V)\right] d E
$$

We assume the sample to have the bare BCS density of states ${ }^{14}$ with the superconducting energy gap $\Delta$ without any smearing or rounding due to inelastic or magnetic pair-breaking scattering mechanism. ${ }^{29}$

$$
\rho_{\mathrm{s}}=\left\{\begin{array}{rcc}
\frac{|E|}{\sqrt{E^{2}-\Delta^{2}}} & : & |E| \geq \Delta \\
0 & : & |E|<\Delta
\end{array}\right.
$$

This assumption results in an upper estimate of the electronic temperature. With the reasonable assumption that the DOS of the probe is constant over the small bias voltage range, the contribution of the probe density of states cancels out upon differentiation of Eq. (1). By numerical integration of

$$
\frac{d I}{d V}(V) \propto \int_{-\pi / 2}^{\pi / 2} \sin \alpha \times I\left(V+\sqrt{2} V_{\bmod } \times \sin \alpha\right) d \alpha
$$

one can take into account the finite modulation voltage $V_{\text {mod }}$ of the lockin amplifier in the description of the measured differential conductance. ${ }^{5}$ 


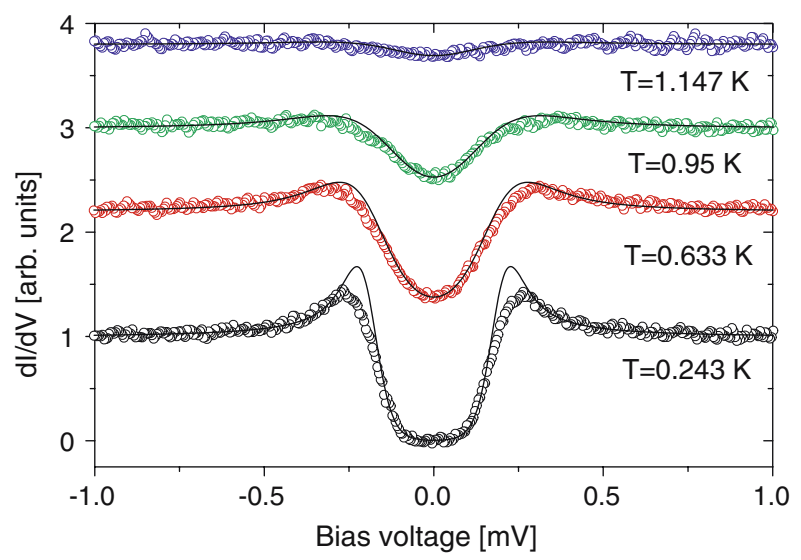

Fig. 2. (Color online) Differential conductance $d I / d V$ of an aluminium tip (sample) measured vs. an evaporated gold film (probe) for different temperatures of the cryostat. The spectra were recorded with a modulation voltage $V_{\mathrm{mod}}=14 \mu \mathrm{V}_{\mathrm{rms}}$ at $T=243 \mathrm{mK}$, and a stabilization current $I=205 \mathrm{pA}$ at $V=4.05 \mathrm{mV}$ has been used. The points are experimental values at different temperatures. Each curve is an average over four measurements per point and recorded within $54 \mathrm{~s}$. The solid lines are fits following Eq. (3) with electron temperatures 0.3, $0.65,0.95$ and $1.18 \mathrm{~K}$. The plots are shifted vertically for clarity.

The best fit is achieved by adjusting the temperature $T_{\mathrm{el}}$ and the superconducting energy gap $\Delta$.

Figure 2 displays the measured differential conductance $d I / d V$ (under the above-mentioned assumptions this is proportional to the DOS) of a superconducting aluminium $(99.999 \%)$ tip versus a gold film measured with a modulation voltage $V_{\text {mod }}=14 \mu \mathrm{V}_{\text {rms }}$. The best fit yields an electron temperature of $T_{\mathrm{el}}=(300 \pm 10) \mathrm{mK}$ and a superconducting gap $\Delta=$ $190 \pm 5 \mu \mathrm{eV}$ for the lowest bath temperature of the cryostat. Our criterion for the best fit is the good description of the region inside the gap and the onset of $d I / d V$ at the gap edge, because smearing due to thermal occupation of quasiparticle states is most prominent at the gap edge. As a result the fit overestimates the $d I / d V$ in the region around the singularity. A better description of that region could be achieved by allowing deviations of the DOS due to pair breaking effects. ${ }^{30}$ However in order to minimize the number of adjustable parameters we did not take that into account and therefore the determined $T_{\mathrm{el}}$ represents an upper limit for the electron temperature. This temperature would correspond to an energy resolution of $\approx 3.5 k_{\mathrm{B}} T_{\mathrm{el}}=90 \mu \mathrm{eV}$. Since the thermometer is located on the ${ }^{3} \mathrm{He}$ bath $\approx 20 \mathrm{~cm}$ above the STM, also a small temperature gradient to 


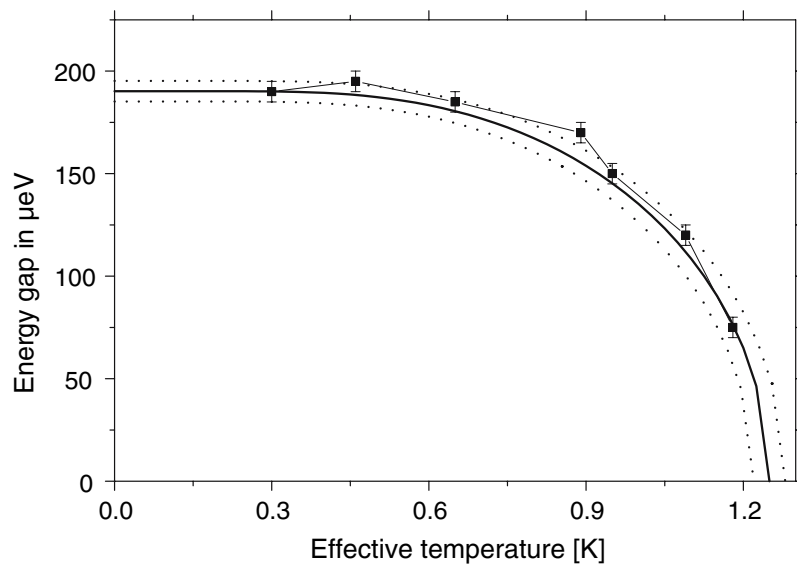

Fig. 3. Evolution of the measured superconducting energy gap of an aluminium tip (sample) at different electronic temperatures. The solid line represents the theoretical curve for $\Delta_{0}=(190 \pm 5) \mu \mathrm{eV}$ and $T_{\mathrm{c}}=1.25 \mathrm{~K}$, the dotted lines correspond to $\Delta_{0}=195 \mu \mathrm{eV}$ and $\Delta_{0}=$ $185 \mu \mathrm{eV}$ and the corresponding $T_{\mathrm{c}}$ according to the BCS theory. The squares are results of the fitting procedure for different electron temperatures. The error bars indicate the accuracy of the fit procedure.

the bath cannot be excluded which would give rise to an enhanced temperature as well.

In order to investigate whether the voltage resolution is limited by the electron temperature or by the lock-in modulation voltage amplitude, which is usually chosen to be smaller or comparable with $20 \mu \mathrm{V}$, we recorded the differential conductance at different temperatures to measure the evaluation of the superconducting energy gap. Figure 3 shows the temperature dependence of the energy gap $\Delta$ and illustrates that we are able to measure changes of the gap of about $20 \mu \mathrm{eV}$. The energy gap for $T=0$ has been determined from the measurement at the lowest temperature and yields $\Delta_{0}=(190 \pm 5) \mu \mathrm{eV}$ and a critical temperature of $T_{\mathrm{c}}=1.25 \mathrm{~K}$. The gap is slightly higher than the literature value for the bulk material but follows the temperature dependence of the BCS theory. ${ }^{14}$ We argue that due to our local measurement and the oxidised surface of the tip, a larger gap is observed as demonstrated in aluminium with oxygen impurities. ${ }^{31}$

For performing systematic studies of the superconducting proximity effect the magnetic field is an important control parameter. When using classical superconductors with small critical field a nonmagnetic and nonsuperconducting setup is thus required. These features are demonstrated by investigating the hysteretic first-order phase transition between the superconducting and normal state of the type I superconductor aluminium. 


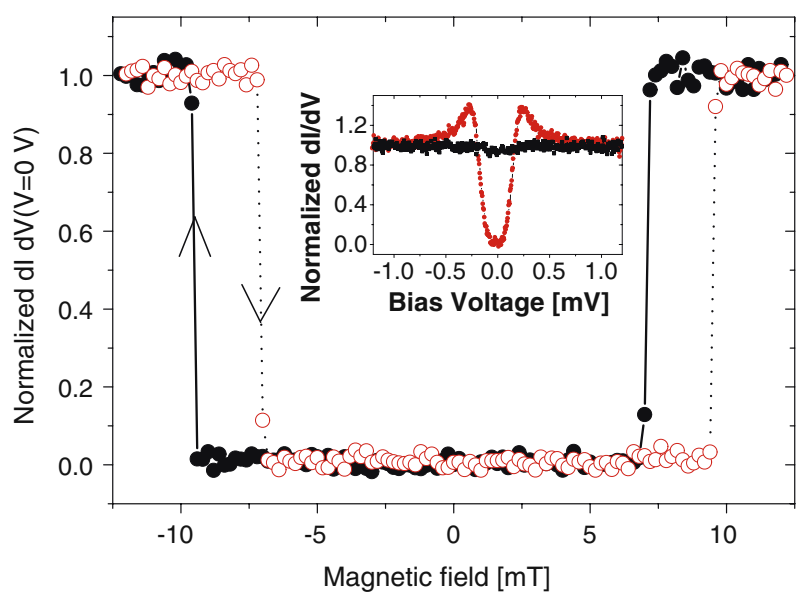

Fig. 4. (Color online) Evolution of the differential conductance in the gap $d I / d V\left(V_{\text {bias }}=0\right)$ versus magnetic field measured at $243 \mathrm{mK}$ with $I=205 \mathrm{pA}$ and a voltage $V=4.05 \mathrm{mV}$. The arrows indicate the sweep direction of the magnetic field enhancing (dashed line) and reducing (solid line) the field. The inset shows the normalized differential conductance for $B=$ $9.4 \mathrm{mT}$ (red curve) below $B_{\mathrm{cl}}$ and for $B=9.5 \mathrm{mT}$ (black curve) above $B_{\mathrm{cl}}$ demonstrating the discontinuous change of the DOS at $B_{\mathrm{cl}}$.

If the external magnetic field exceeds the critical field $B_{\mathrm{c} 1}$ the superconducting state becomes energetically unfavorable and the external magnetic field penetrates the superconductor. The DOS thus changes abruptly from the superconducting to the normal one as shown in the inset of Fig. 4. When reducing the external field below $B_{\mathrm{c} 1}$ in a clean system, the superconducting phase does not nucleate immediately, and the normal phase will be "super cooled" down to the lower critical field $B_{\mathrm{c} 2}$. The latter is related to the coherence length $\xi$ via $B_{\mathrm{c} 2} \approx \phi_{0} / \xi^{2}$, where $\phi_{0}$ is the superconducting flux quantum $h / 2 e .{ }^{29}$

We use an aluminium tip (99.999\%) that has been cut from a wire with diameter $1 \mathrm{~mm}$ and a thin evaporated gold film. For minimizing the demagnetization factor ${ }^{29}$ the magnetic field is applied in $z$-direction parallel to the superconducting tip. The transition is monitored by sweeping the magnetic field and recording the differential conductance at zero bias $d I / d V\left(V_{\text {bias }}=0\right)$. Conductance values $d I / d V\left(V_{\text {bias }}=0\right)$ close to zero indicate the superconducting phase while a large $d I / d V\left(V_{\text {bias }}=0\right)$ signals the normalconducting phase (see inset of Fig. 4). At a measuring temperature of $243 \mathrm{mK}$ we observe an electron temperature of $T_{\mathrm{el}}=300 \mathrm{mK}$ and a critical field of $B_{\mathrm{cl}}=9.5 \mathrm{mT}$. The critical field is slightly below the expected value for the observed electron temperature of the tip. ${ }^{32}$ For decreasing 


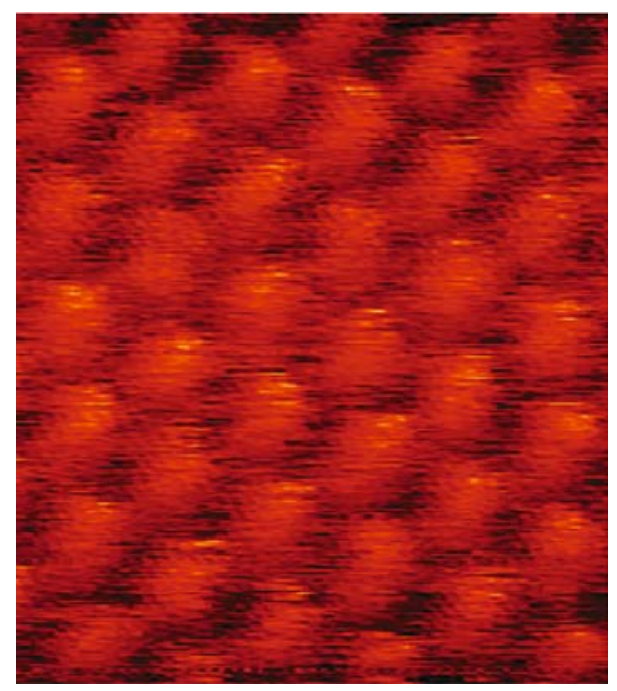

Fig. 5. (Color online) Unfiltered topographic image of HOPG measured at $258 \mathrm{mK}$ and $1 \mathrm{~T}$ $(U=0.62 \mathrm{~V}, I=690 \mathrm{pA})$ with a copper slider, showing atomic resolution. The image size is $1.3 \times 1.3 \mathrm{~nm}^{2}$.

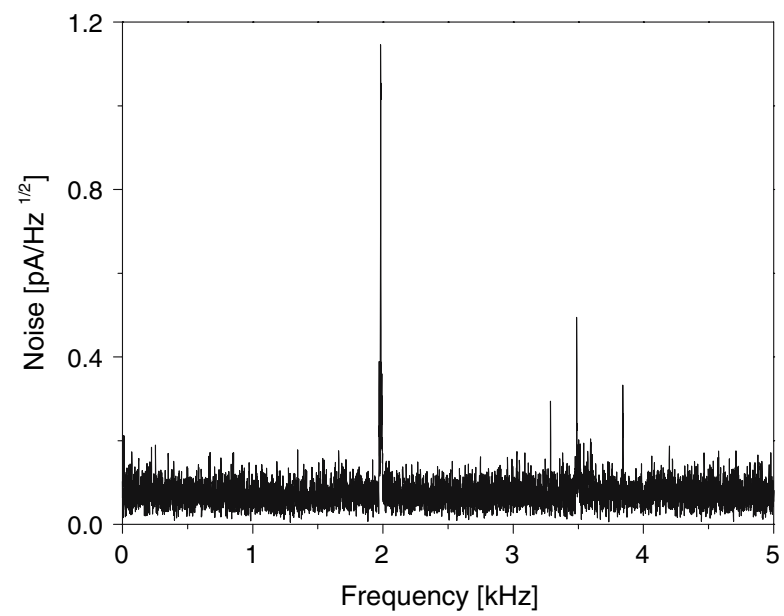

Fig. 6. Current noise spectrum of the STM measured at $243 \mathrm{mK}$ with a tungsten tip versus an evaporated $50 \mathrm{~nm}$ gold surface on aluminium $(V=4.06 \mathrm{mV} \mathrm{V}, I=403 \mathrm{pA})$. The feedback was turned off. The most prominent resonance at $2 \mathrm{kHz}$ is of mechanical origin. 
fields we clearly see the supercooling effect of the normal phase down to $7.5 \mathrm{mT}$ and $-7.5 \mathrm{mT}$, respectively, corresponding to a coherence length of $\xi \approx 540 \mathrm{~nm}$. The symmetry of the curve demonstrates the absence of parasitic magnetic fields and the ability of the system to act reliably at low fields. When using type II superconductors with high critical fields rather large external fields have to be applied. As a second experiment, imaging of HOPG proofs the successful operation in high magnetic fields. Although the STM is not designed for topographic measurements with high spatial resolution it achieves atomic resolution on HOPG at $T=$ $258 \mathrm{mK}$ without and with a magnetic field of $B=1 \mathrm{~T}$, which is our largest measuring field (see Fig. 5). From the concept and the chosen materials we expect the STM to work at even higher magnetic fields. From the calibration of the topographic image with atomic resolution, we can roughly estimate the maximum scan range at very low temperatures to be $4.3 \mu \mathrm{m}$. Due to piezo nonlinearities the real scan range might be different. The overall noise level and the stability are shown in Fig. 6. The currentnoise spectrum has been taken while the feedback loop has been turned off in order to avoid oscillations by the feedback loop. As can be seen there are no resonances below $\approx 2 \mathrm{kHz}$, a fact which is important for imaging. The mechanical origin of the peak just around $2 \mathrm{kHz}$ has been verified by external excitation. For all other resonances (above $3 \mathrm{kHz}$ ) the reason could not be revealed unambiguously. Nevertheless, the presence of those resonances does not hinder the atomic resolution.

\section{SUMMARY}

A small, compact and rigid STM has been built up. It achieves atomic resolution on HOPG even in the presence of vibrations in the $\mathrm{kHz}$ frequency range. Important for spectroscopy on superconductors is the achieved high energy resolution, which has been demonstrated on superconducting aluminium. By measuring the critical field of a superconducting aluminium tip and demonstrating atomic resolution at very low temperatures and in an external magnetic field of $1 \mathrm{~T}$ we proof that the STM is nonmagnetic.

\section{ACKNOWLEDGMENTS}

The authors wish to thank G. Goll, C. Sürgers and G. Wittig for helpful discussions about technical and operational STM challenges and U. Schröter for the numerical evaluation. We thank $M$. Wolz for his contribution to this work. The work is supported by the Landesstiftung 
Baden-Württemberg within the framework of the Kompetenznetz "Funktionelle Nanostrukturen".

\section{REFERENCES}

1. M. Kugler, Ch. Renner, Ø. Fischer, V. Mikheev, and G. Batey, Rev. Sci. Instrum. 71, 1475 (2000).

2. T. Matsui, H. Kambara, and H. Fukuyama, J. Low. Temp. Phys. 121803 (2000).

3. T. Matsui, H. Kambara, I. Ueda, T. Shishido, Y. Miyatake, and H. Fukuyama, Physica B 329, 1653 (2003).

4. A. J. Heinrich, C. P. Lutz, J. A. Gupta, and D. M. Eigler, AIP Conf. Proc. 696, 100 (2003).

5. J. Wiebe, A. Wachowiak, F. Meier, D. Haude, T. Foster, M. Morgenstern, and R. Wiesendanger, Rev. Sci. Instrum. 75, 4871 (2004).

6. P. Davidsson, H. Olin, M. Persson, and S. Pehrson, Ultramicroscopy 42-44, 1470 (1992).

7. S. H. Pan, E. W. Hudson, and J. C. Davis, Rev. Sci. Instrum. 70, 1459 (1999).

8. N. Moussy, H. Courtois, and B. Pannetier, Rev. Sci. Instrum. 72, 128 (2001).

9. H. Suderow, S. Vieira, J. D. Strand, S. Bud'ko, and P. C. Canfield, Phys. Rev. B 69, 060504(R) (2004).

10. A. K. Gupta, L. Crétinon, N. Moussy, B. Pannetier, and H. Courtois, Phys. Rev. B 69, 104514 (2004).

11. N. Moussy, H. Courtois, and B. Pannetier, Europhys. Lett. 55, 861 (2001).

12. W. Escoffier, C. Chapelier, and F. Lefloch, Phys. Rev. B. 72, 140502 (2005).

13. W. Belzig, C. Bruder, and G. Schön, Phys. Rev. B 54, 9443 (1996).

14. J. Bardeen, L. N. Cooper, and J. R. Schrieffer, Phys. Rev. 108, 1175 (1957).

15. A. Habenicht, M. Olapinski, F. Burmeister, P. Leiderer, and J. Boneberg Science 309, 2043 (2005).

16. EBL\#2 EBL Products Inc. 91 Prestige Park Circle, East Hartford, CT 06108, USA.

17. Macor is a registered trademark of Corning, Inc., One Riverfront Plaza, Corning, NY 14831, USA.

18. G. Nunes Jr. and Dinsie Williams, J. Vac. Technol. B 13, 1063 (1995).

19. F. Mugele, A. Rettenberger, J. Boneberg, and P. Leiderer, Rev. Sci. Instrum. 69, 1765 (1998).

20. S. Kleindiek and K. H. Herrmann, Rev. Sci. Instrum. 64, 692 (1993).

21. Ch. Renner, Ph. Niedermann, A. D. Kent, and Ø. Fischer, Rev. Sci. Instrum. 61, 965 (1990).

22. Type UT20-SS-SS made by Micro-Coax, 206 Jones Blvd. Pottstown, PA 19464-3465, USA.

23. D. C. Glattli, P. Jacques, A. Kumar, P. Pari, and L. Saminadayar, J. Appl. Phys. 81, 7350 (1997).

24. M. Nittmann, P. Ziemann, W. Buckel, and G. Linker, Z. Phys. B Condens. Matter 41, 205 (1981).

25. J. M. Martinis, M. H. Devoret, and J. Clarke, Phys. Rev. B 35, 4682 (1987).

26. C. Strunk private communication

27. M. Tinkham, 2nd edition of Introduction to Superconductivity, Mc. GrawHill (1996).

28. S. Caplan and G. Chalin, Phys. Rev. 138, A1428, (1965).

29. J. Tersoff and D. R. Hamann, Phys. Rev. B 31, 805 (1985).

30. J. Bardeen, Phys. Rev. Lett. 6, 57 (1961).

31. RHK Technology Inc., 1050 East Maple Road, Troy, MI 48083, USA.

32. S. Skalski, O. Betbeder-Matibet, and P. R. Weiss, Phys. Rev. 136, A1500 (1964). 